\title{
INVENTARIO DE REPRESENTACIONES DE LA VIDA ES SUEÑO
}

\section{PERFORMANCE INVENTORY OF LIFE IS A DREAM}

\author{
José Manuel TRIVES PÉREZ \\ IES Juan de Villanueva (Madrid) \\ lolotrives@yahoo.es
}

Agradecimientos

a Víctor Infantes de Miguel y José Romera Castillo, sin cuyos apoyos no existiría este trabajo.

Resumen: Se trata de un artículo bibliográfico en el que se recogen cuantas representaciones se han encontrado registradas de La vida es sueño y se ordenan cronológicamente indicando, además del año, el lugar y la fuente bibliográfica.

Abstract: This article is a bibliographic study that consists in a performance list of La vida es sueño from its days until ours for all over the pla- 
ces where it has been performed. All items are named by the place and the date of the performance, and they also show the source where it was taken.

Palabras clave: Cartelera. La vida es sueño. Calderón de la Barca.

Key words: Listings. Life is a dream. Calderón de la Barca.

\section{INTRODUCCIÓN}

La tabla que presentamos a continuación es el primer intento de reunir cuantas representaciones de La vida es sueño se han registrado sin barrera ninguna de tiempo ni de espacio. Nos preceden no pocos trabajos que se interesaron por la cartelera de alguna localidad puntual o por algún autor en concreto, pero ninguno que, más allá de la aproximación (Díez Borque y Peláez Martín, 2000; Mancebo, 2002; Romera Castillo, 2011: 141-183) ${ }^{1}$, abordara monográficamente un título en particular, ni por supuesto, La vida es sueño en particular. Recogemos más de medio millar de registros, lo que supera con creces cualquier compilación previa, pero eso no nos hace olvidar que aún más de las que son son las que nos faltan.

Estamos convencidos de que la primera representación que detallamos no puede ser ni de lejos el estreno de La vida es sueño y que los catorce registros conservados en todo el siglo XVII bien pudieron superarse con anterioridad al festejo fonsaldiño citado, pues allá, ya lo llevaba alguna fama según se desprende de los documentos conservados del jurista García de Albertos (Davis y Varey, 2003: I, 33.34). Allí queda escrito que La vida es sueño era el único título requerido expresamente de entre los contratados a la compañía de Antonio Piñero.

Hemos también de advertir que nuestra búsqueda no ha llegado más allá de la biblioteca y que no ha pisado archivo ni hemeroteca salvo en muy contadas ocasiones y casi más por capricho que por erudición. Por ello somos conscientes de no engrosar la lista de representaciones con

${ }^{1}$ El trabajo de Díez Borque y Peláez Martín aborda la trayectoria escénica de un autor (Pedro Calderón de la Barca) en un siglo (el siglo XX); el de Yolanda Mancebo se queda en propuesta de lo que aquí pretendemos continuar nosotros; y, finalmente, en el capítulo señalado de José Romera encontrará el lector el punto de partida para el estudio de la historia escénica de distintos autores (del siglo de Oro y otros) en general (véase el capítulo 4 donde además encontrará una perspectiva escénica de nuestra obra en algunas ciudades y durante el siglo XIX: pp. 154-156), de Lope de Vega en particular (véase el capítulo 5,pp. 173-184) y sobre el teatro áureo español llevado a las pantallas de cine, televisión y otros medios (véase capítulo 6, pp. 185-199). 
demasiados hallazgos sobresalientes. Antes hemos preferido emplear nuestras fuerzas en reunir todo el vocerío que hacen los libros hablando de teatro calderoniano en nuestras bibliotecas y hacer que se respeten el turno de palabra y nos cuenten de uno en uno lo registrado sobre las representaciones de La vida es sueño.

Pero el que se pone a enumerar estrenos cae en el error de confundir, en una entrada de igual valor, la temporada de una compañía en su teatro estable y sus múltiples funciones con la representación singular de una gira teatral que volverá a tener nueva entrada como nuevo estreno en la siguiente localidad donde le lleve su itinerario. Hubiera sido más apropiado titular el trabajo como un listado de estrenos si no fuera porque la palabra estreno no deja de ser menos controvertida que ninguna otra. Nosotros enumeramos cada una de las veces que La vida es sueño ha estrenado alguna plaza y las puestas en escena que, aun siendo representadas en la misma sala y el mismo año, se distancian por más de un día. Éste es el motivo de que en nuestra lista parezcan repetidas algunas entradas como las de París, 1742, Madrid, 1735 o Almagro, 1984, por ejemplo. Recogemos en diferentes entradas las representaciones realizadas en días no consecutivos. Quizá no sea la mejor manera de valorar fielmente la realidad. Simplemente damos cuenta de nuestra opción con el ánimo de poder ser mejor entendidos.

En cuanto a la documentación que conservamos de las representaciones hay que decir que, por lo general, describe las puestas en escena de manera muy desigual. Mientras de algunas conservamos información detallada de la compañía, de la sala, de la crítica, de los beneficios, etc., de otras apenas tenemos una referencia imprecisa. Existe otra mucha información de interés sobre la mayoría de ellas, pero sobre el papel seguro que se haría farragoso. Lo mismo opino en cuanto a la bibliografía. Expongo únicamente las referencias de las fuentes citadas. A toda la consultada le encontraremos mejor sitio en otro lugar.

Nuestro listado reduce sus registros al lugar y fecha del estreno, y a la referencia documental, la cual ajustamos breve y concisa a las normas editoriales de SIGNA. Sin embargo, algunas escapan a lo establecido mereciendo una explicación. Cuando la fuente es Internet procuramos la dirección http vinculada a su acceso directo. En el caso de información inédita obtenida directamente desde la fuente remitimos a la institución que nos proporciona la documentación. Dos son apenas las excepciones incluidas en este apartado, «Centro de Documentación Teatral» (CDT) y la productora teatral «Pérez de la Fuente» (Producciones «Pérez de la Fuente»). 
Sé que el trabajo tiene no pocas deficiencias, pues tratando de cartelera, uno se equivoca más veces de las que acierta y acaba encontrando unos hallazgos que no admiten estadística frente a sus faltas. Pero también queda el buen sabor de boca de ponerle nombre al primer mojón del camino, a la primera cartelera histórica de La vida es sueño. Así pues será un orgullo ver corregido y completado este trabajo pionero que nace enclenque como todos nacimos pero que esperamos crezca y sea fecundo.

No quisiera cerrar esta introducción sin agradecer a SELITEN@T y, de manera especial, a su director José Romera Castillo, su importantísima contribución al estudio de la cartelera durante sus ya más de veinte años de historia y el espacio que aquí nos brindan para dar luz a nuestro trabajo. Al resto de los citados en nuestra bibliografía, que con sus trabajos han contribuido a esta biografía escénica de La vida es sueño, por sus registros, muchas gracias.

\section{LISTADO DE REPRESENTACIONES DE LA VIDA ES SUEÑO}

1. Fuente el Saz, 1636.

Davis y Varey (2003: I, 33-34;

II, 721).

2. Bruselas, 1647.

Baczynska (1991: 24).

3. Ámsterdam, 1654.

Sullivan (1998: 64).

4. Hamburgo, 1654.

Sullivan (1998: 64).

5. Ámsterdam, 1658.

Sullivan (1998: 64).

6. Luneburgo, 1666.

Baczynska (1991: 24).
7. Madrid, 1673.

Subirats (1977: 479)

8. Dresde, 1674.

Franzbach (1982: 229).

9. Madrid, 1684.

Varey y Shergold (1989: 23940).

10. Lima, 1684.

Arenz (2000: 27).

11. Valladolid, 1688.

Alonso (1923: 307).

12. Torgau, 1690.

Franzbach (1982: 16, 230). 
13. Valladolid, 1691.

Alonso (1923: 307).

14. Hamburgo, 1693.

Sullivan (1998: 105 y ss.)

15. Magdeburgo, 1693.

Sullivan (1998: 107).

16. Valladolid, 1694.

Alonso (1923: 312).

17. Madrid, 1695.

Subirats (1977: 479).

18. Wernigerode, c.a. 1700.

Franzbach (1982: 230-31).

19. Guadalajara (Méjico, 1702).

Arenz (2000: 226).

20. Valladolid, 1705.

Alonso (1923: 338).

21. Lima, 1708.

Hesse (1955: 20).

22. Madrid, 1709.

Andioc (2008: 890).

23. Madrid, 1710.

Andioc (2008: 890).

24. Madrid, 1714.

Andioc (2008: 890).

25. Madrid, 1714.

Andioc (2008: 890).

26. Madrid, 1715.

Andioc (2008: 890).
27. Madrid, 1716.

Andioc (2008: 890).

28. París, 1717.

Franzbach (1982: 46).

29. París, 1717.

Franzbach (1982: 46).

30. Guatemala, 1717.

Hesse (1955:18).

31. Madrid, 1717.

Andioc (2008: 890).

32. Madrid, 1718.

Andioc (2008: 890).

33. Madrid, 1718.

Andioc (2008: 890).

34. París, 1718.

Franzbach (1982: 46).

35. París, 1718.

Franzbach (1982: 46).

36. París, 1718.

Franzbach (1982: 46).

37. Madrid, 1719.

Andioc (2008: 890).

38. Madrid, 1719.

Andioc (2008: 890).

39. Riga, 1719.

Sullivan, 1998, p. 97.

40. Madrid, 1720.

Andioc (2008: 890). 
41. Hamburgo, 1721.

Franzbach (1982: 231).

42. Madrid, 1722.

Andioc (2008: 890).

43. Madrid, 1722.

Andioc (2008: 890).

44. París, 1722.

Franzbach (1982: 46).

45. París, 1722.

Franzbach (1982: 46).

46. París, 1723.

Franzbach (1982: 46).

47. Madrid, 1724.

Andioc (2008: 890).

48. París, 1725.

Franzbach (1982: 46).

49. París, 1725.

Franzbach (1982: 46).

50. París, 1725.

Franzbach (1982: 46).

51. Valladolid, 1725.

Alonso (1923: 347).

52. París, 1726.

Franzbach (1982: 46).

53. París, 1726.

Franzbach (1982: 46).

54. Valladolid, 1726.

Alonso (1923: 349).
55. Madrid, 1726.

Andioc (2008: 890).

56. París, 1728.

Franzbach (1982: 46).

57. Madrid, 1728.

Andioc (2008: 890).

58. Madrid, 1728.

Andioc (2008: 890).

59. Madrid, 1728.

Andioc (2008: 890).

60. Valladolid, 1728.

Alonso (1923: 352).

61. Barcelona, 1730.

Par (1929: 333).

62. Barcelona, 1731.

Par (1929:334).

63. Madrid, 1731.

Andioc (2008: 890).

64. Madrid, 1731.

Andioc (2008: 890).

65. Lima 1731,

Hesse (1955: 22).

66. París, 1732.

Franzbach (1982: 46).

67. París, 1732.

Franzbach (1982: 46).

68. París, 1732.

Franzbach (1982: 46). 
69. París, 1732.

Franzbach (1982: 46).

70. París, 1732.

Franzbach (1982: 46).

71. París, 1732.

Franzbach (1982: 46).

72. París, 1732.

Franzbach (1982: 46).

73. Valladolid, 1732.

Alonso (1923: 359).

74. París, 1733.

Franzbach (1982: 46).

75. París, 1733.

Franzbach (1982: 46).

76. París, 1733.

Franzbach (1982: 46).

77. Madrid, 1734.

Andioc (2008: 890).

78. Madrid, 1735.

Andioc (2008: 890).

79. Madrid, 1735.

Andioc (2008: 890).

80. Madrid, 1737.

Andioc (2008: 890).

81. Valladolid, 1737.

Alonso (1923: 366).

82. Madrid, 1739.

Andioc (2008: 890).
83. Hamburgo, 1740.

Franzbach (1982: 231).

84. Valladolid, 1740.

Alonso (1923: 369).

85. Francfort, 1741.

Franzbach (1982: 232).

86. Valladolid, 1741.

Alonso (1923: 371).

87. Valladolid, 1742.

Alonso (1923: 374).

88. París, 1742.

Franzbach (1982: 46).

89. París, 1742.

Franzbach (1982: 46).

90. París, 1742.

Franzbach (1982: 46).

91. París, 1742.

Franzbach (1982: 46).

92. París, 1742.

Franzbach (1982: 46).

93. París, 1742.

Franzbach (1982: 46).

94. París, 1742.

Franzbach (1982: 46).

95. París, 1742.

Franzbach (1982: 46).

96. París, 1744.

Franzbach (1982: 47). 
97. París, 1745.

Franzbach (1982: 47).

98. París, 1745.

Franzbach (1982: 47).

99. París, 1745.

Franzbach (1982: 47).

100. París, 1745.

Franzbach (1982: 47).

101. Madrid, 1746.

Andioc (2008: 890).

102. París, 1746.

Franzbach (1982: 46).

103. París, 1746.

Franzbach (1982: 46).

104. París, 1746.

Franzbach (1982: 46).

105. París, 1746.

Franzbach (1982: 46).

106. Buenos Aires, 1746.

Arenz (2000: 227).

107. París, 1747.

Franzbach (1982: 46).

108. París, 1747.

Franzbach (1982: 46).

109. París, 1747.

Franzbach (1982: 46).

110. París, 1747.

Franzbach (1982: 46).
111. Madrid, 1748.

Andioc (2008: 890).

112. París, 1748.

Franzbach (1982: 46).

113. París, 1748.

Franzbach (1982: 46).

114. París, 1748.

Franzbach (1982: 46).

115. París, 1748.

Franzbach (1982: 46).

116. París, 1749.

Franzbach (1982: 46).

117. París, 1750.

Franzbach (1982: 46).

118. París, 1750.

Franzbach (1982: 46).

119. París, 1750.

Franzbach (1982: 46).

120. París, 1750.

Franzbach (1982: 47).

121. París, 1750.

Franzbach (1982: 47).

122. Valladolid, 1750.

Alonso (1923: 385).

123. París, 1751.

Franzbach (1982: 47).

124. París, 1751.

Franzbach (1982: 47). 
125. Valladolid, 1751.

Alonso (1923: 388).

126. París, 1752.

Franzbach (1982: 46).

127. París, 1752.

Franzbach (1982: 46).

128. París, 1753.

Franzbach (1982: 46).

129. París, 1754.

Franzbach (1982: 46).

130. París, 1754.

Franzbach (1982: 46).

131. Alemania, 1754.

Franzbach (1982: 233).

132. París, 1755.

Franzbach (1982: 46).

133. París, 1755.

Franzbach (1982: 46).

134. Valladolid, 1756.

Alonso (1923: 392).

135. Valladolid, 1756.

Alonso (1923: 395).

136. París, 1756.

Franzbach (1982: 46).

137. París, 1756.

Franzbach (1982: 46).

138. París, 1757.

Franzbach (1982: 46).
139. París, 1757.

Franzbach (1982: 46).

140. París, 1758.

Franzbach (1982: 46).

141. París, 1758.

Franzbach (1982: 46).

142. Madrid, 1758.

Andioc (2008: 890).

143. París, 1759.

Franzbach (1982: 46).

144. Madrid, 1759.

Andioc (2008: 890).

145. París, 1760.

Franzbach (1982: 46).

146. París, 1760.

Franzbach (1982: 46).

147. París, 1760.

Franzbach (1982: 47).

148. Viena, 1760.

Franzbach (1982: 53).

149. Nuremberg, 1760.

Franzbach (1982: 233).

150. París, 1761.

Franzbach (1982: 47).

151. París, 1761.

Franzbach (1982: 47).

152. Madrid, 1763.

Andioc (2008: 890). 
153. Toledo, 1763.

Montero (1942: 433).

154. Toledo, 1763.

Montero (1942: 435).

155. Madrid, 1764.

Andioc (2008: 890).

156. Valladolid, 1764.

Alonso (1923: 397).

157. Madrid, 1765.

Andioc (2008: 890).

158. Toledo, 1765.

Montero (1942: 438).

159. Madrid, 1766.

Andioc (2008: 890).

160. Madrid, 1766.

Andioc (2008: 890).

161. Toledo, 1768.

Montero (1942: 443).

162. Madrid, 1770.

Andioc (2008: 890).

163. Madrid, 1770,

Andioc (2008: 890).

164. Toledo, 1771.

Montero (1942: 445).

165. Sevilla, 1771.

Aguilar (1974: 288).

166. Sevilla, 1771.

Aguilar (1974: 288).
167. Sevilla, 1772.

Aguilar (1974: 288).

168. Sevilla, 1772.

Aguilar (1974: 288).

169. Toledo, 1772.

Montero (1942: 447).

170. Toledo, 1773.

Montero (1942: 450).

171. Sevilla, 1773.

Aguilar (1974: 288).

172. Barcelona, 1774.

Par (1929: 336).

173. Sevilla, 1775.

Aguilar (1974: 288).

174. México, 1775.

Hesse (1955: 23).

175. Sevilla, 1775.

Aguilar (1974: 288).

176. Barcelona, 1775.

Par (1929: 338).

177. Madrid, 1775.

Andioc (2008: 890).

178. Madrid, 1775.

Andioc (2008: 890).

179. Toledo, 1775.

Montero (1942: 452).

180. Sevilla, 1776.

Aguilar (1974: 288). 
181. Barcelona, 1777.

Par (1929: 340).

182. Sevilla, 1778.

Aguilar (1974: 288).

183. Barcelona, 1778.

Par (1929: 342).

184. México, 1778.

Hesse (1955: 24).

185. Madrid, 1778.

Andioc (2008: 890).

186. Madrid, 1778.

Andioc (2008: 890).

187. Valladolid, 1778.

Alonso (1923: 403).

188. Barcelona, 1779.

Par (1929: 344).

189. Valladolid, h.1780.

Alonso (1923: 404).

190. Gijón, 1781.

Menéndez (1981: 92).

191. Madrid, 1782.

Andioc (2008: 890).

192. Madrid, 1782.

Andioc (2008: 890).

193. Barcelona, 1784.

Par (1929: 500).

194. Barcelona, 1785.

Par (1929: 504).
195. México, 1785.

Hesse (1955: 24).

196. Madrid, 1785.

Coe (1935: 231).

197. Madrid, 1786.

Andioc (2008: 890).

198. París, 1787.

Franzbach (1982: 47).

199. Barcelona, 1787.

Par (1929: 509).

200. Barcelona, 1787.

Par (1929: 511).

201. Madrid, 1787.

Coe (1935: 231).

202. Valladolid, 1787.

Alonso (1923: 406).

203. Madrid, 1788.

Coe (1935: 231).

204. Barcelona, 1789.

Par (1929: 596).

205. México, 1790.

Hesse (1955: 24).

206. México, 1790.

Hesse (1955: 24).

207. México, 1791.

Hesse (1955: 25).

208. Barcelona, 1792.

Par (1929: 606). 
209. México, 1792.

Hesse (1955: 25).

210. Madrid, 1792.

Coe (1935: 231).

211. Buenos Aires, a. 1792.

Ordaz (1946: 17).

212. Barcelona, 1793.

Par (1929: 608).

213. Madrid, 1795.

Cotarelo (1902: 513).

214. Valencia, 1796.

Zabala (1982: 326).

215. Sevilla, 1800.

Aguilar (1968:45).

216. Barcelona, 1802.

Suero (1987: 64).

217. Buenos Aires, 1804.

Hesse (1955: 27).

218. Madrid, 1810.

Cotarelo (1902: 536).

219. Sevilla, 1811.

Aguilar (1968: 294).

220. Madrid, 1811.

Cotarelo (1902: 536).

221. Barcelona, 1812.

Suero (1987: 364).

222. Weimar, 1812.

Durán (1983: 59).
223. Barcelona, 1813.

Suero (1987: 385).

224. Mallorca, 1813.

Larraz (1974: 354).

225. Madrid, 1814.

Cotarelo (1902: 543).

226. Barcelona, 1814.

Suero (1987: 420).

227. Barcelona, 1814.

Suero (1987: 420).

228. Madrid, 1815.

Lorenz (1938: 330).

229. Madrid, 1815.

Lorenz (1938: 330).

230. Madrid, 1816.

Cotarelo (1902: 547).

231. Buenos Aires, 1816.

Arenz (2000: 228).

232. Barcelona, 1817.

Suero (1987: III, 63-64).

233. Madrid, 1818.

Cotarelo (1902: 550).

234. Barcelona, 1820.

Suero (1987: III, 185).

235. Madrid, 1820.

Adams (1936: 354).

236. Barcelona, 1822.

Suero Roca (1987: III, 244) 
237. Valladolid, 1824.

Alonso (1949: 45).

238. Lwow, 1826.

Baczynska (1991: 26).

239. Sevilla, 1826.

Aguilar (1968: 45).

240. Barcelona, 1826.

Suero (1987: III, 358).

241. Barcelona, 1827.

Suero (1987: III, 389).

242. Madrid, 1832.

Simón (1961: 26).

243. San Juan (Puerto Rico), 1833.

Hesse (1955: 18).

244. Madrid, 1833.

Simón (1961: 28).

245. Madrid, 1834.

Adams (1926: 254).

246. Sevilla, 1834.

Aguilar (1968: 45).

247. Madrid, 1835.

Simón (1961: 38).

248. Madrid, 1835.

Simón (1961: 39).

249. Madrid, 1835.

Simón (1961: 40).

250. Sevilla, 1835.
Aguilar (1968: 45).

251. Sevilla, 1836.

Aguilar (1968: 45).

252. Buenos Aires, 1837.

Arenz (2000: 228-229).

253. Madrid, 1842.

Herrero (1963: 92).

254. Madrid, 1842.

Herrero (1963: 92).

255. Madrid, 1843.

Herrero (1963: 92).

256. Madrid, 1844.

Herrero (1963: 92).

257. Madrid, 1844.

Herrero (1963: 92).

258. Madrid, 1844.

Herrero (1963: 92).

259. Madrid, 1845.

Mancebo (2002: 98).

260. Madrid, 1846.

Herrero (1963: 92).

261. Madrid, 1847.

Adams (1936: 354).

262. Madrid, 1848.

Herrero (1963: 92).

263. Madrid, 1849.

Herrero (1963: 92).

264. Madrid, 1856. 
Simón (1963: 213).

265. Barcelona, 1857.

Cervelló (http://www.uned.es /centro-investigacion-SELITEN@T/pdf/CARLOS_CERVELL_(BARCELONA).pdf ).

266. Bolonia, 1862.

Cattaneo (2000: 295).

267. Bolonia, 1869.

Cattaneo (2000: 295).

268. Badajoz, 1872.

Suárez (http://www.uned.es/centro-investigacion-SELITEN@T lexcel/Badajoz_Su\%C3\%Alrez\% 20Mu\%C3\%Bloz.xls).

269. Málaga, 1872-73.

Pino (1985: 358).

270. Madrid, 1874.

Simón (1963: 215).

271. Madrid, 1875.

Simón (1963: 215).

272. Málaga, 1875.

Pino (1985: 362).

273. Madrid, 1876.

Simón (1963: 216).

274. Madrid, 1876.

Simón (1963: 216).

275. Valladolid, 1876.

Alonso (1947: 167).
276. Valladolid, 1877.

Alonso (1947: 172).

277. Málaga, 1877-78.

Pino (1985: 366).

278. Madrid, 1879.

Simón (1963: 216).

279. Madrid, 1879.

Simón (1963: 216).

280. Madrid, 1879.

Simón (1963: 217).

281. Valencia, 1880.

Simón (1963: 217).

282. México, 1880.

Arenz (2000: 226.

283. Madrid, 1881.

Simón (1963: 218).

284. Madrid, 1881

Simón (1963: 221).

285. Madrid, 1881

Simón (1963: 221).

286. Jerez, 1881.

Álvarez (http://www.uned.es /centro-investigacion-SELITEN@T/excel/CarteleraTeatralJerezana.xls).

Álvarez (2010: 184).

287. León, 1881.

Fernández (http://www.uned.es /centro-investigacion-SELI- 
TEN@T/excel/Leon_Estefania_Fernandez.xls).

Aragón (2006b: 40)

288. León, 1882.

Fernández (http://www.uned.es /centro-investigacion-SELITEN@T/excel/Leon_Estefania_Fernandez.xls).

Romera (2003: 374).

Aragón (2006b: 40).

289. León, 1883.

Fernández (http://www.uned.es /centro-investigacion-SELITEN@T/excel/Leon_Estefania_Fernandez.xls).

290. Madrid, 1883.

Simón (1963: 223).

291. Madrid, 1885.

Simón (1963: 223).

292. Badajoz, 1886.

Suárez (http://www.uned.es/centro-investigacion-SELITEN@T lexcel/Badajoz_Su\%C3\%Alrez\% 20Mu\%C3\%Bloz.xls).

293. Toledo, 1886.

Torres (http://www.uned.es/centro-investigacion-SELITEN@T /excel/Toledo_Torres\%20Lara.xl s).

294. Madrid, 1888.

Simón (1963: 223).

295. Málaga, 1889.
Pino (1985: 484).

296. Logroño, 1891.

Benito (http://www.uned.es/centro-investigacion-SELITEN@ T/excel/inma.xls)

Aragón (2006b: 47-48).

297. León, 1893.

$\begin{array}{lllllllll} & \mathrm{e} & \mathrm{r} & \mathrm{n} & \mathbf{a} & \mathrm{n} & \mathrm{d} & \mathrm{e} & \mathrm{z}\end{array}$ (http://www.uned.es/centro-investigacion-SELITEN@T/excel/Leon_Estefania_Fernandez.xls).

298. Madrid, 1893.

Simón (1963: 224).

299. Jerez 1894.

Álvarez (http://www.uned.es /centro-investigacion-SELITEN@T/excel/CarteleraTeatralJerezana.xls).

300. Jerez, 1894.

Álvarez (http://www.uned.es /centro-investigacion-SELITEN@T/excel/CarteleraTeatralJerezana.xls).

Álvarez (2010: 192).

301. Bruselas, 1894.

Simón (1963: 224).

302. Las Palmas, 1895.

López (http://www.uned.es/centro-investigacion-SELITEN@

T/excel/Las_Palmas_de_Gran_C anaria_Mar_Lopez.xls). 
Aragón (2006b: 78).

303. Madrid, 1897.

Simón (1963: 224).

304. Logroño, 1898.

Benito (http://www.uned.es/centro-investigacion-SELITEN@T lexcel/inma.xls)

Aragón (2006b: 64).

305. Toledo, 1898.

Torres (http://www.uned.es/centro-investigacion-SELITEN@ T/excel/Toledo_Torres\%20Lara. $x l s)$.

306. Oviedo, 1899.

Arrones (1993: 77).

307. Oviedo, 1901.

Arrones (1993: 85).

308. Logroño, 1902

Somalo (http://www.uned.es/ centro -investigacion-SELITEN@T/pdf/SomaloFernandez.pdf).

Aragón (2006b: 163).

Somalo (2006: 502).

309. Madrid, 1905.

Mancebo (2002: 92).

310. Pontevedra, 1908.

Aparicio (http://www.uned.es /centro-investigacion-SELITEN@T/excel/Pontevedra_PAparicio.xls).
Aragón (2006b: 107-108).

311. Viena, 1910.

Díez y Peláez (2000: 384).

312. Moscú, 1912.

Monforte (2008: 74).

313. Madrid, 1913.

Andura (2000: 124).

314. Logroño, 1915.

Somalo (http://www.uned.es/centro-investigacion-SELITE N@T/pdf/SomaloFernandez.pdf).

Aragón (2006b: 163).

Somalo (2006: 523).

315. Moscú, 1915.

Monforte (2008: 74).

316. Pontevedra, 1916.

Aparicio (http://www.uned.es /centro-investigacion-SELITEN@T/excel/Pontevedra_PAparicio.xls).

317. Madrid, 1917.

Díez y Peláez (2000: 384).

318. Madrid, 1918.

Andura (2000: 124).

319. Madrid, 1918.

Andura (2000: 124).

320. Madrid, 1919.

Díez y Peláez (2000: 384).

321. Madrid, 1920. 
Dougherty y Vilches (2000: 476).

322. Madrid, 1920.

Dougherty y Vilches (2000: 476).

323. Madrid, 1921.

Díez y Peláez (2000: 384).

324. Albacete, 1921.

Linares (http://www.uned.es/centro-investigacion-SELITEN@T lexcel/Albacete_Linares_1901_1923.xls).

325. Madrid, 1923.

Dougherty y Vilches (2000: 476).

326. París, 1922.

Díez y Peláez (2000: 384).

327. Albacete, 1923.

Linares (http://www.uned.es/centro-investigacion-SELITEN@T lexcel/Albacete_Linares_1901_1923.xls).

328. Cambridge, 1925.

García (2003: 183).

329. Madrid, 1926.

Andura (2000:125).

330. Londres, 1926.

Díez y Peláez (2000: 384).

331. París, 1927.

Díez y Peláez (2000: 384).
332. Austria, 1927.

Díez y Peláez (2000: 384).

333. Segovia, 1928.

González (http://www.uned.es /centro-investigacion-SELITEN@T/excel/paloma\%20segovia.xls)

Aragón (2006b: 131-32).

334. Madrid, 1928.

Díez y Peláez (2000: 384).

335. Logroño, 1929.

Somalo (http://www.uned.es/centro-investigacion-SELITEN@T /pdf/SomaloFernandez.pdf).

Aragón (2006b: 163).

336. Madrid, 1929.

Díez y Peláez (2000: 384).

337. Cambridge, 1929.

García (2003: 186).

338. Albacete, 1929.

Ochando (http://www.uned.es /centro-investigacion-SELITEN@T/excel/Albace te_1924_1936_Ochando.xls).

Aragón (2006b: 150-151).

339. Albacete, 1930.

Ochando (http://www.uned.es /centro-investigacion-SELITEN@T/excel/Albace te_1924_1936_Ochando.xls).

Aragón (2006b: 152-153). 
340. Madrid, 1930.

Díez y Peláez (2000: 384).

341. Madrid, 1931.

McGaha (1979: 15).

342. Madrid, 1931.

González (1996: 107)

343. Düsseldorf, 1931.

Díez y Peláez (2000: 384).

344. Madrid, 1932.

González (1996: 107)

345. Madrid, 1932.

McGaha (1979: 21)

346. Dresde, 1933.

Díez y Peláez (2000: 384).

347. Madrid, 1933.

González (1996: 271)

348. Madrid, 1934.

González (1996: 402)

349. Segovia, 1934.

González (http://www.uned.es /centro-investigacion-SELITEN@T/excel/paloma\%20segovia.xls)

Aragón (2006b: 139).

350. Berlín, 1934.

London (1998: 150).

351. Madrid, 1935.

González (1996: 467)

352. Madrid, 1936.
Andura (2000:127).

353. Cambridge, 1936.

García (2003: 188).

354. Buenos Aires, 1936.

Díez y Peláez (2000: 384).

355. Lwow (Polonia), 1937.

Díez y Peláez (2000: 384).

356. Madrid, 1938.

González (1996: 548)

357. Logroño, 1939.

Somalo (http://www.uned.es/centro-investig a c ionSELITEN@T/pdf/SomaloFernandez.pdf).

Aragón (2006b: 163).

358. Albacete, 1939.

Ochando (http://www.uned.es /centro-investigacion-SELITEN@T/excel/Albace te_1924_1936_Ochando.xls).

359. Madrid, 1940.

García y Muñoz (2001: 420).

360. Madrid, 1940.

García y Muñoz (2001: 420).

361. Italia, 1940.

Díez y Peláez (2000: 384).

362. Chile, 40

Reverte (2008: 162).

363. Madrid, 1942. 
García y Muñoz (2001: 420).

364. Viena, 1942.

Díez y Peláez (2000: 384).

365. Barcelona, 1943.

Aznar (1994: 20)

366. Madrid, 1943.

CDT

367. Berlín, 1943.

Díez y Peláez (2000: 384).

368. Nápoles, 1943.

García (2006: 335)

369. El Dueso, 1944.

Díez y Peláez (2000: 384).

370. Madrid, 1944.

Andura (2000:131).

371. Granada, 1944.

Díez y Peláez (2000: 384).

372. Francia, 1944.

Díez y Peláez (2000: 384).

373. Valencia, 1947.

Sirera (1993: 117)

374. México, 1947.

Díez y Peláez (2000: 384).

375. Viena, 1948.

Díez y Peláez (2000: 384).

376. Madrid, 1949.

García y Muñoz (2001: 421).

377. México, 1950.
Díez y Peláez (2000: 384).

378. Madrid, 1950.

García y Muñoz (2001: 421).

379. Baden-Baden, 1950

Díez y Peláez (2000: 384).

380. Oviedo, 1952.

Arrones (1993: 233).

381. Düsseldorf, 1952.

Díez y Peláez (2000: 384).

382. Madrid, 1953.

García y Muñoz (2001: 422).

383. Hamburgo, 1953.

Díez y Peláez (2000: 384).

384. París, 1954.

Andura (2000:135).

385. Madrid, 1955.

Díez y Peláez (2000: 384).

386. Oviedo, 1957.

Arrones (1993: 247).

387. Mannhein, 1956.

Díez y Peláez (2000: 384).

388. Guadalajara (México), 1956.

Cerda (http://www.uned.es/centro-investigacion-SELITEN@T lexcel/Guadalajara_.xls). 389. Madrid, 1956.

García y Muñoz (2001: 421).

390. Madrid, 1957. 
Díez y Peláez (2000: 384).

391. Viena, 1957.

Díez y Peláez (2000: 384).

392. Bochum, 1957.

Díez y Peláez (2000: 384).

393. Madrid, 1958.

Díez y Peláez (2000: 384).

394. Berlín, 1959.

Díez y Peláez (2000: 384).

395. Stuttgart, 1960.

Díez y Peláez (2000: 384).

396. Madrid, 1960.

Díez y Peláez (2000: 385).

397. Montauban, 1961.

Díez y Peláez (2000: 385).

398. Valencia, 1962.

Alcalde (1993: 128).

399. Lisboa, 1962.

Díez y Peláez (2000: 385).

400. Barcelona, 1963.

Díez y Peláez (2000: 385).

401. Guadalajara (México), 1964.

Cerda (http://www.uned.es/centro-investigacion-SELITEN@T lexcel/Guadalajara_.xls).

402. Barcelona, 1965.

Díez y Peláez (2000: 385).

403. Viena, 1965.
Díez y Peláez (2000: 385).

404. Dusseldorf, 1965.

Díez y Peláez (2000: 385).

405. Stuttgart, 1965.

Díez y Peláez (2000: 385).

406. México D.F., 1965.

Díez y Peláez (2000: 385).

407. Wroclaw, 196.

Díez y Peláez (2000: 385).

408. Madrid, 1968.

García y Muñoz (2001: 425)

409. Varsovia, 1969.

Díez y Peláez (2000: 385).

410. Cracovia, 1969.

Díez y Peláez (2000: 385).

411. Barcelona, 1969.

Díez y Peláez (2000: 385).

412. Schwäbisch Hall, 1969.

Díez y Peláez (2000: 385).

413. Madrid, 1969-70.

García y Muñoz (2001: 425).

414. Madrid, 1969-70.

García y Muñoz (2001: 425).

415. Madrid, 1970.

García y Muñoz (2001: 426)

416. Madrid, 1970.

García y Muñoz (2001: 426)

417. Alcalá de Henares, 1970. 
Aragón (http://www.uned.es/ centro-investigacion-SELITEN@T/excel/Alcala_Irene.xls).

418. Italia, 1970.

Díez y Peláez (2000: 385).

419. New York, 1971.

Díez y Peláez (2000: 384).

420. Madrid, 1973.

García y Muñoz (2001: 426).

421. Londres, 1973.

Díez y Peláez (2000: 385).

422. Londres, 1973.

Díez y Peláez (2000: 385).

423. Frankfurt, 1973.

Díez y Peláez (2000: 385).

424. Bamberg, 1973.

Díez y Peláez (2000: 385).

425. Torino, 1975.

Huerta (2008: 82).

426. Milán, 1975.

Huerta (2008: 82).

427. Brescia, 1975-76.

Huerta (2008: 82).

428. Madrid, 1976.

García y Muñoz (2001: 426).

429. Brescia, 1978.

Huerta (2008: 82)

430. Forchtenstein, 1978.
Huerta (2008: 63).

431. Francia, 1978.

Huerta (2008: 71).

432. Tolouse, 1979

Huerta (2008: 71).

433. Milán, 1980.

Huerta (2008: 82)

434. Miami, 1980.

Huerta (2008: 67)

435. Madrid, 1981.

García y Muñoz (2001: 428)

436. Madrid, 1981.

García y Muñoz (2001: 428)

437. Las Palmas, 1981.

Díez y Peláez (2000: 385).

438. Granada, 1981.

Díez y Peláez (2000: 385).

439. Nueva York, 1981.

Huerta (2008: 67).

440. North Caroline, 1981.

Huerta (2008: 67).

441. Viena, 1982.

Gimber, 2008, pp. 52-53.

442. Berlín, 1982.

Gimber, 2008, pp. 52-53.

443. Dusseldorf, 1982.

Huerta (2008: 63).

444. Bulgaria, 1982. 
Dimitrova (2002: 467).

445. París, 1982.

Huerta (2008: 71).

446. Francia, 1983.

Huerta (2008: 71).

447. Polonia, 1983.

Huerta (2008: 83).

448. Stratford-on-Avon, 1983.

Huerta (2008: 73).

449. Viena, 1983.

Huerta (2008: 64).

450. Forchtestein 1984.

Huerta (2008: 64).

451. Valladolid, 1984.

Dimitrova (2002: 467).

452. Almagro 1984.

Díez y Peláez (2000: 385).

453. Sofia, 1984.

Huerta (2008: 64)

454. Almagro, 1984.

Díez y Peláez (2000: 385).

455. Zaragoza, 1984.

Dimitrova (2002: 467).

456. Alicante, 1984.

García (1992: 266).

457. Alicante, 1984.

García (1992: 273).

458. Londres, 1984.
Huerta (2008: 73)

459. Nürnberg, 1984.

Huerta (2008: 64)

460. Berlín, 1985.

Huerta (2008: 64)

461. Varsovia, 1985.

Huerta (2008: 83).

462. Polonia, 1985.

Huerta (2008: 84)

463. Italia, 1985.

Huerta (2008: 82)

464. Palermo, 1985.

Huerta (2008: 82)

465. Palermo, 1985.

Huerta (2008: 82)

466. Almagro, 1985.

Díez y Peláez (2000: 385).

467. Munich 1985.

Huerta (2008: 64)

468. USA, 1985.

Huerta (2008: 68)

469. Berlín, 1986.

Huerta (2008: 64)

470. Montevideo, 1986.

Huerta (2008: 77).

471. Lisboa, 1986.

Huerta (2008: 84)

472. Hungría, 1986. 
Huerta (2008: 81).

473. Caspe, 1987.

Díez y Peláez (2000: 385).

474. Sofía, 1987.

Díez y Peláez (2000: 385).

475. California, 1987.

Huerta (2008: 68)

476. Portugal, 1987.

Huerta (2008: 84)

477. Lisboa, 1988.

Huerta (2008: 84).

478. Sujumi, 1988.

Huerta (2008: 86)

479. Moscú, 1988.

Siliunas (2000: 188).

480. Palermo, 1988.

Huerta (2008: 82)

481. Varsovia, 1988.

Sabik (2000: 216).

482. Guadalajara (México), 1988.

Cerda (http://www.uned.es/centro-investigacion-SELITEN@T lexcel/Guadalajara_.xls).

483. Guadalajara (México), 1989.

Cerda (http://www.uned.es/centro-investigacion-SELITEN@T/excel/Guadalajara_.xls).
484. Munich, 1989.

Huerta (2008: 64).

485. Cambridge, 1989.

Huerta (2008: 68)

486. Francia, 1989.

Huerta (2008: 72)

487. Inglaterra, 1989.

Huerta (2008: 74)

488. Londres, 1989.

Huerta (2008: 74)

489. Colombia, 1989 .

Huerta (2008: 77).

490. Chihuahua, 1989 .

Huerta (2008: 77).

491. Nueva York, 1989.

Huerta (2008: 68)

492. Solothurn, 1990.

Huerta (2008: 64)

493. Hamburgo, 1990.

Huerta (2008: 64)

494. Karlsruhe, 1990.

Huerta (2008: 64)

495. Chicago, 1990.

Huerta (2008: 68)

496. Francia, 1991.

Huerta (2008: 72)

497. Venecia, 1991.

Huerta (2008: 82) 
498. Chile, 1991.

Huerta (2008: 78).

499. Landshut, 1992.

Arnscheidt (2003: 496).

500. Roma, 1992.

Díez y Peláez (2000: 385).

501. Leeds, 1992.

Díez y Peláez (2000: 385).

502. Tbiblisi, 1992.

Huerta (2008: 86)

503. Almería, 1992.

García y Muñoz (2001: 429).

504. Huesca, 1992.

Díez y Peláez (2000: 385).

505. Portalegre, 1992.

Huerta (2008: 84).

506. El Escorial, 1992.

Díez y Peláez (2000: 385).

507. París, 1992.

Díez y Peláez (2000: 385).

508. Washington, 1992.

Huerta (2008: 68)

509. Braunschweig, 1993.

Huerta (2008: 64)-65

510. Londres, 1993.

Díez y Peláez (2000: 386).

511. Lima, 1994.

Huerta (2008: 78).
512. São Paulo, 1994.

Rodrigues (2003: 405).

513. Palma, 1994.

Díez y Peláez (2000: 386).

514. Cáceres, 1995.

http://www.granteatrocc.com/clasico/95/

515. Nueva York, 1995.

Huerta (2008: 69)

516. Greifswald, 1995.

Huerta (2008: 65)

517. Heilbronn, 1995.

Arnscheidt (2003: 497).

518. Milán, 1995.

Huerta (2008: 82)

519. Wuppertal, 1996.

Huerta (2008: 65).

520. Almagro, 1996.

Díez y Peláez (2000: 386).

521. Segovia, 1996.

Díez y Peláez (2000: 386).

522. Cádiz, 1996.

Díez y Peláez (2000: 386).

523. Cáceres, 1997.

http://www.granteatrocc.com/clasico/97/vida.html

524. Valladolid, 1997.

CDT. 
525. Londres, 1997.

Huerta (2008: 74)

526. Londres, 1997.

Huerta (2008: 74)

527. Basilea, 1997.

Huerta (2008: 65)

528. Moscú, 1997.

Huerta (2008: 86).

529. Tolosa, 1998.

Aragón (2003: 222).

530. Dresde, 1998.

Arnscheidt (2003: 499).

531. Murcia, 1998.

Díez y Peláez (2000: 386).

532. Sao Paolo, 1998.

Huerta (2008: 86).

533. Río de Janeiro, 1998.

Huerta (2008: 86)

534. USA, 1998.

Huerta (2008: 69)

535. Denver, 1998.

Huerta Calvo, 2008, p. 69

536. Edimburgo, 1998.

Huerta (2008: 74)

537. UK, 1998.

Díez y Peláez (2000: 386).

538. Moscú, 1998.

Huerta (2008: 86)
539. San Sebastián de los Reyes, 1999.

Romera (2000: 41).

540. Chicago, 1999.

Schizzano, 2003

541. Texas, 1999.

Huerta (2008: 69)

542. Dortmund, 1999.

Huerta (2008: 65).

543. Viena, 1999.

Huerta (2008: 65).

544. Salzburgo, 1999.

Huerta (2008: 65).

545. Polonia, 1999.

Díez y Peláez (2000: 386).

546. Buenos Aires, 1999.

Huerta (2008: 79)

547. Londres, 1999.

Huerta (2008: 75).

548. Río de Janeiro, 2000.

Huerta (2008: 84).

549. Curitiba, 2000.

Huerta (2008: 84).

550. Washington, 2000.

Huerta (2008: 69).

551. Chicago, 2000.

Huerta (2008: 69)

552. Madrid, 2000. 
Díez y Peláez (2000: 386).

553. Marsella, 2000.

Huerta (2008: 73)

554. Milán, 2000.

Huerta (2008: 83)

555. Krefeld, 2000.

Huerta (2008: 66).

556. Burgos, 2000.

CDT

557. Ronda, 2000.

CDT

558. Alcalá de Henares, 2001.

CDT

559. Oregon, 2001.

Huerta (2008: 70).

560. Frankfurt, 2001.

Huerta (2008: 66)

561. Los Ángeles, 2001.

Huerta (2008: 70).

562. Nueva York, 2001.

Huerta (2008: 70)

563. Illinois, 2001.

Huerta (2008: 70)

564. Filadelfia, 2001 .

Huerta (2008: 70)

565. Narbona, 2001.

Huerta (2008: 73)

566. Venezuela, 2001.
Huerta (2008: 79)

567. Japón, 2002.

Huerta (2008: 83).

568. Curitiba, Brasil, 2002

Huerta (2008: 84).

569. Nueva York, 2003.

Huerta (2008: 70).

570. Madrid, 2003.

CDT

571. Orlowo, 2003.

Huerta (2008: 84).

572. Badajoz, 2005.

CDT

573. Cáceres, 2006.

http://www.granteatrocc.com/clasico/06/08.html

574. Gladbeck, 2006.

Huerta (2008: 66)

575. USA, 2006 .

Huerta (2008: 71).

576. USA, 2007.

Huerta (2008: 71).

577. Rió de Janeiro, 2007.

http://riodejaneiro.cervantes.es/FichasCultura/Ficha41680_55 _1.htm

578. Bogotá, 2008.

http://www.mincultura.gov.co/?id categoria $=8497$ 
579. Inglaterra, 2008. .

Huerta (2008: 75)

580. Alcalá de Henares, 2008.

Producciones «Pérez de la Fuente».

581. San Lorenzo de El Escorial, 2008.

Producciones «Pérez de la Fuente».

582. Niebla, 2008.

Producciones «Pérez de la Fuente».

583. Berlín, 2008.

Producciones «Pérez de la Fuente».

584. Barcelona, 2008.

Producciones «Pérez de la Fuente».

585. Torrelodones, 2008.

Producciones «Pérez de la Fuente».

586. Palma de Gran Canarias, 2008.

Producciones «Pérez de la Fuente».

587. Orense, 2008.

Producciones «Pérez de la Fuente».

588. Alcobendas, 2008.

Producciones «Pérez de la Fuente».
589. Torrejón de Ardoz, 2008.

Producciones «Pérez de la Fuente».

590. Móstoles, 2008.

Producciones «Pérez de la Fuente».

591. Madrid, 2008.

Producciones «Pérez de la Fuente».

592. Toledo, 2009.

Producciones «Pérez de la Fuente».

593. Santander, 2009.

Producciones «Pérez de la Fuente».

594. Fuenlabrada, 2009.

Producciones «Pérez de la Fuente».

595. Lugo, 2009.

Producciones «Pérez de la Fuente».

596. Velilla de San Antonio, 2009.

Producciones «Pérez de la Fuente».

597. Valencia, 2009.

Producciones «Pérez de la Fuente».

598. Getafe, 2009.

Producciones «Pérez de la Fuente». 
599. Salamanca, 2009.

Producciones «Pérez de la Fuente».

600. Segovia, 2009.

Producciones «Pérez de la Fuente».

601. Las Palmas de Gran Canaria, 2009.

Producciones «Pérez de la Fuente».

602. Las Rozas, 2009.

Producciones «Pérez de la Fuente».

603. Cáceres, 2009.

Producciones «Pérez de la Fuente».

604. Chinchilla de Montearagón, 2009.

Producciones «Pérez de la Fuente».

605. Olmedo, 2009.

Producciones «Pérez de la Fuente».

606. Valladolid, 2009.

Producciones «Pérez de la Fuente».

607. Valdepeñas, 2009.

Producciones «Pérez de la Fuente».

608. Milán, 2009.
Producciones «Pérez de la Fuente».

609. Guadalajara, 2009.

Producciones «Pérez de la Fuente».

610. Alcázar de San Juan, 2009.

Producciones «Pérez de la Fuente».

611. Soria, 2009.

Producciones «Pérez de la Fuente».

612. Manzanares, 2009.

Producciones «Pérez de la Fuente».

613. Alcázar de San Juan, 2010. http://tu.tv/videos/la-vida-es-sueno_1_1

614. Torredelcampo (Jaén), 2010.

http://www.torredelcampo.es/index.php?option $=$ com_jcalpro\&It emid $=123 \&$ extmode $=$ view\&extid $=961$

615. Bogotá, 2010.

http://www.youtube.com/watch?v $=k 7 \mathrm{WZvvlAbrc} \&$ feature $=$ related

616. Bogotá, 2010.

www.youtube.com/watch? $v=U t$ Lesmdt9A

617. Buenos Aires, 2010.

http://www.cscomunicarte.com.a r/la-vida-es-sueno-con-joaquin- 
furriel-y-muriel-santa-ana-en-elteatro-san-martin/

618. Buenos Aires, 2010. http://www.deboralachter.com.ar /ver_nota.asp?codigo_nota $=439$ 619. Buenos Aires, 2011. http://www.entradateatro.com.ar/ tag/la-vida-es-sueno-2011/ 620. Granada, 2011. http://secretariageneral.ugr.es/ pages/tablon/*/noticias-canalugr/2011/03/23/representacionteatral-de-arapsodia-na-2-lavida-es-sueano

621. Almagro, 2011.

http://lacuevadelrio.blogspot.com /2011/07/gira-la-vida-es-sueno2011.html

622. Ballesteros de Calatrava, 2011.

http://lacuevadelrio.blogspot.com /2011/07/gira-la-vida-es-sueno2011.html

623. Socuéllamos, 2011.

http://lacuevadelrio.blogspot.com /2011/07/gira-la-vida-es-sueno2011.html

624. Almuradiel, 2011.

http://lacuevadelrio.blogspot.com /2011/07/gira-la-vida-es-sueno2011.html

625. Alhambra, 2011. http://lacuevadelrio.blogspot.com /2011/07/gira-la-vida-es-sueno2011.html

626. Poblete, 2011.

http://lacuevadelrio.blogspot.com /2011/07/gira-la-vida-es-sueno2011.html

627. Albaladejos, 2011.

http://lacuevadelrio.blogspot.com /2011/07/gira-la-vida-es-sueno2011.html

628. Picón, 2011.

http://lacuevadelrio.blogspot.com /2011/07/gira-la-vida-es-sueno2011.html

629. Corral de Calatrava, 2011.

http://lacuevadelrio.blogspot.com /2011/07/gira-la-vida-es-sueno2011.html

630. La Solana, 2011.

http://lacuevadelrio.blogspot.com /2011/07/gira-la-vida-es-sueno2011.html

631. Chillón, 2011.

http://lacuevadelrio.blogspot.com /2011/07/gira-la-vida-es-sueno2011.html

632. Aldea del Rey, 2011.

http://lacuevadelrio.blogspot.com /2011/07/gira-la-vida-es-sueno2011.html

633. Alcoba, 2011. 
http://lacuevadelrio.blogspot.com /2011/07/gira-la-vida-es-sueno2011.html

634. Llanos del Castillo, 2011. http://lacuevadelrio.blogspot.com /2011/07/gira-la-vida-es-sueno2011.html

635. Puebla de Don Rodrigo, 2011.

http://lacuevadelrio.blogspot.com 12011/07/gira-la-vida-es-sueno2011.html

636. Argamasilla de Alba, 2011. http://lacuevadelrio.blogspot.com /2011/07/gira-la-vida-es-sueno2011.html

637. Almadenejos, 2011.

http://lacuevadelrio.blogspot.com /2011/07/gira-la-vida-es-sueno2011.html

638. El torno, 2011.

http://lacuevadelrio.blogspot.com /2011/07/gira-la-vida-es-sueno2011.html

639. Brazatortas, 2011.

http://lacuevadelrio.blogspot.com /2011/07/gira-la-vida-es-sueno2011.html

640. Terrinches, 2011.

http://lacuevadelrio.blogspot.com /2011/07/gira-la-vida-es-sueno2011.html

641. Cabezarrubias, 2011. http://lacuevadelrio.blogspot.com /2011/07/gira-la-vida-es-sueno2011.html

642. Guadalmez, 2011.

http://lacuevadelrio.blogspot.com /2011/07/gira-la-vida-es-sueno2011.html

643. Villarubia de los Ojos, 2011. http://lacuevadelrio.blogspot.com /2011/07/gira-la-vida-es-sueno2011.html

644. Fuenllana, 2011.

http://lacuevadelrio.blogspot.com /2011/07/gira-la-vida-es-sueno2011.html

645. Torralba de Calatrava, 2011. http://lacuevadelrio.blogspot.com /2011/07/gira-la-vida-es-sueno2011.html

646. Cabezarados, 2011.

http://lacuevadelrio.blogspot.com 12011/07/gira-la-vida-es-sueno2011.html

647. Granátula de Calatrava, 2011.

http://lacuevadelrio.blogspot.com 12011/07/gira-la-vida-es-sueno2011.html

648. Infantes, 2011.

http://lacuevadelrio.blogspot.com /2011/07/gira-la-vida-es-sueno2011.html

649. Arenales, 2011. 
http://lacuevadelrio.blogspot.com /2011/07/gira-la-vida-es-sueno2011.html

650. Retuerta, 2011.

http://lacuevadelrio.blogspot.com /2011/07/gira-la-vida-es-sueno2011.html
651. Buenos Aires, 2012.

http://www.complejoteatral.gob.ar /htm/obras/sueno0.html

652. Almagro, 2012.

http://www.abc.es/20111215/cult u ra/abci-portillo201112151625.html

\section{REFERENCIAS BIBLIOGRÁFICAS}

ADAMS, N. B. (1936). «Siglo de Oro Plays in Madrid: 1820-1850». Hispanic Review 4, 342-357.

- (1926). «Notes on Spanish Plays at the Beginning of the Romantic Period». Romanic Review 17, 128-142.

AGUILAR PIÑAL, F. (1968). Cartelera prerromántica sevillana: años 1800-1836. Madrid: CSIC.

- (1974). Sevilla y el teatro en el siglo XVIII. Oviedo: Cátedra Feijoo.

ALCALDE CRESPO, G. (1993). «El teatro Club: Antonio García Fernando y Tomás Abad». En 60 anys de teatre universitari, M. Aznar, N. Diago y M. ${ }^{a}$ Mancebo (com. y coord.), 122-29. Valencia: Universitat de València.

ALONSO CORTÉS, N. (1923). El teatro en Valladolid. Madrid: [s.n.].

- (1947). El teatro en Valladolid (s. XIX). Valladolid: Imprenta Castellana.

ÁLVAREZ HORTIGOSA, F. (2009). Historia del teatro en Jerez de la Frontera durante la segunda mitad del siglo XIX (Tesis realizada bajo la dirección de Alberto Ramos Santana y María E. Cantos Casenave y defendida en la Universidad de Cádiz, en 2009. Puede consultarse en http://www.uned.es/centro-investigacion-SELITEN@T/estudios_sobre_teatro.html).

- (2010). «Puestas en escena del teatro clásico español del siglo de oro y del siglo XVIII en Jerez de la Frontera (1852-1900)». Signa. Revista de la Asociación Española de Semiótica 19, 182-212. 
ANDIOC, R. y COULON, M. (2008). Cartelera teatral madrileña del siglo XVIII: 1708-1808. Madrid: Fundación Universitaria Española.

ANDURA VARELA, F. (2000). «Calderón en la escena española, 19002000». En Calderón en escena: siglo XX, J.M. . Díez y Andrés Peláez (eds.), 123-156. Madrid: T. F. Artes Gráficas, D. L.

APARICIO MORENO, P. (2002). La vida escénica en Pontevedra: 1901 1924 (Tesis realizada bajo la dirección de M. ${ }^{a}$ Pilar Espín Templado y defendida en la UNED, en septiembre de 2000. Puede consultarse en http://www.uned.es/centro-investigacion-SELITEN@T/estudios_sobre_teatro.html. Extracto incluido en Aragón, 2006b)

ARAGÓN GONZÁLEZ, I. (2003). «El teatro español en la cartelera de Toulouse entre 1995 y 2002». Teatro (Revista de Estudios Teatrales) 19, 197-256.

- (2006). La vida escénica en Alcalá de Henares 1939-1982, (Tesis realizada bajo la dirección de José Romera. Puede consultarse en http://www.uned.es/centro-investigacion-SELITEN@T/estudios_sobre_teatro.html.

ARAGÓN GONZÁLEZ, I. (coord.) (2006b) «Puestas en escena de nuestro teatro áureo en algunas ciudades españolas durante los siglos XIX y XX». Signa. Revista de la Asociación Española de Semiótica 15, 11186.

ARENZ, S. (2000). «Algunas notas sobre la presencia de Pedro Calderón de la Barca en Hispanoamérica». En Calderón en escena: siglo XX, J.M. ${ }^{a}$ Díez y Andrés Peláez (eds.), 225-40. Madrid: T. F. Artes Gráficas, D. L.

ARNSCHEIDT, G. y PARDELLAS VELAY, R. (2003) «Calderón en la escena de habla alemana (1990-2001)». En Teatro Calderoniano sobre el tablado, M. Tietz (ed.), 493-499. Sttutgart: Steiner.

ARRONES PEÓN, L. (1993). Teatro Campoamor: Crónica de un coliseo centenario, Oviedo 1892-1992. Oviedo: Real Instituto de Estudios Asturianos.

AZNAR SOLER, M. y CASARES I ROCA, T. (1994). El teatre universitari a Barcelona. Barcelona: UAB.

BACZYNSKA, B. (1991). «La recepción de La vida es sueño en Polonia». Castilla: Boletín del Departamento de Literatura Española 16, 1938. 
BENITO ARGÁIZ, I. (2003). La vida escénica en Logroño (1850-1900). (Tesis realizada bajo la dirección de M. ${ }^{\text {a }}$ Pilar Espín Templado y Miguel Ángel Muro Munilla y defendida en la Universidad de La Rioja, en 2003. Puede consultarse en http://www.uned.es/centro-investigacion-SELITEN@T/estudios_sobre_teatro.html. Extracto incluido en Aragón, 2006b)

CATTANEO, M. (2000). «Representaciones calderonianas en Italia en el siglo XX». En Calderón en escena: siglo XX, J.M. ${ }^{a}$ Díez y Andrés Peláez (eds.), 191-210. Madrid: T. F. Artes Gráficas, D. L.

CASSOL, A. (2002). «Una adaptación inédita de «La vida es sueño»: «La vita humana è un sogno» de Andrea Bendinelli». En Calderón 2000. Homenaje a Kurt Reichenberger en su 80 cumpleaños, I. Arellano (ed.), II, 445-55. Kassel: Reichenberger.

CERDA MUÑOS, A. (1999). La actividad escénica en Guadalajara (México): 1920-1990 (Tesis realizada bajo la dirección de José Romera Castillo, defendida en la UNED, en septiembre de 1999. Pueden consultarse tesis y cartelera en http://www.uned.es/centro-investigacion-SELITEN@T/estudios_sobre_teatro.html).

CERVELLÓ ESPAÑOL, C. (2009). La vida escénica en Barcelona 1855 1865 (Teatro Principal y Teatro Circo Barcelonés) (Tesis realizada bajo la dirección de M. ${ }^{a}$ Pilar Espín Templado y defendida en la UNED, en febrero de 2009. Puede consultarse en http://www.uned.es/centro-investigacion-SELITEN@T/estudios_sobre_teatro.html).

COE, A. (1935). Catálogo bibliográfico y crítico de las comedias anunciadas en los periódicos de Madrid, desde 1661 hasta 1819. Baltimore: John Hopkins University.

COTARELO Y MORI, E. (1902). Isidoro Máiquez y el teatro de su tiempo. Madrid: Imprenta de José Perales y Martínez.

COTTICELLI, F. (2002). «Sobre Calderón y « La vida es sueño»: momentos del teatro contemporáneo». En Calderón 2000. Homenaje a Kurt Reichenberger en su 80 cumpleaños, I. Arellano (ed.), 457-66. Kassel: Reichenberger.

DAVIS, Ch. y VAREY, J.E. (2003). Actividad teatral en la región de Madrid según los protocolos de Juan García de Albertos, 1634-1660: estudio y documentos. London: Tamesis. 
DÍEZ BORQUE, J. M. a y PELÁEZ MARTÍN, A. eds.(2000). Calderón en escena: siglo XX. Madrid: T. F. Artes Gráficas, D. L.

DIMITROVA, M. (2002). «Tiempo y espacio en La vida es sueño: su funcionamiento en una puesta en escena búlgara». En Calderón 2000. Homenaje a Kurt Reichenberger en su 80 cumpleaños, I. Arellano (ed.), II, 467-73. Kassel: Reichenberger.

DURÁN, M. y GONZÁLEZ ECHEVARRÍA, R. (1976). Calderón y la crítica. Madrid: Gredos.

FERNÁNDEZ GARCÍA, E. (1997). León y su actividad escénica en la segunda mitad del siglo XIX (Tesis realizada bajo la dirección de José Romera Castillo y defendida en la UNED, en junio de 1997. Puede consultarse en http://www.uned.es/centro-investigacion-SELITEN@T lestudios_sobre_teatro.html. Extracto incluido en Romera, 2003 y Aragón, 2006b).

FRANZBACH, M. (1982). El teatro de Calderón en Europa. Madrid: Fundación Universitaria Española.

GARCÍA FERRÓN, E.; ROS BERENGUER, C. y ARACIL VARÓN, B. (1992). Cartelera teatral de Alicante, 1975-1991. Alicante: Secretariado de Publicaciones de la Universidad.

GARCÍA GÓMEZ, Á. M. (2003). «Contextualización de las primeras puestas en escena de «La vida es sueño» $(1925,1929)$ en Inglaterra dentro del marco de la crítica anglo-irlandesa del siglo XIX». En Teatro Calderoniano sobre el tablado, M. Tietz (ed.), 185-204. Stuttgart: Steiner.

GARCÍA LORENZO, L. (2006). «La vida es sueño» y la ópera: «Life is a dream» de Lewis Spratlan y James Maraniss (1975-1978) En «El Siglo de Oro en escena». Homenaje a Marc Vitse, O. Gorsse y F. Serralta (ed.), 335-49. [Toulouse]: Presses Universitaires du Mirail.

- y MUÑOZ CARABANTES, M. (2001). «El teatro de Calderón en la escena española (1939-1999)». En Calderón desde el 2000, J.M. a Díez Borque (ed.), 419-33. Madrid: Ollero y Ramos.

GONZÁLEZ, L. M. (1996). «La escena madrileña durante la II República, (1931-1939)». Teatro. Revista de Estudios Escénicos 9/10, 7-627.

GONZÁLEZ BLANCH, P. (2004). El teatro en Segovia (1918-1936) (Tesis realizada bajo la dirección de José Romera y defendida en la 
UNED, noviembre de 2004. Puede consultarse en http://www.uned.es/centro-investigacion-SELITEN@T/estudios_sobre_teatro.html. Extracto incluido en Aragón, 2006b).

HERRERO SALGADO, F. (1961). Cartelera teatral madrileña II: años 1840-1849. Madrid: CSIC.

HESSE, E.W. (1955). «Calderon’s Popularity in the Spanish Indies». Hispanic Review 23, 12-27.

HUERTA CALVO, J. (2008). «La vuelta al mundo de nuestros clásicos». Cuadernos de Teatro Clásico 24, I, 35-87.

LARRAZ, E. (1974). «Le théâtre à Palma de Majorque pendant la Guerre de 1’Independance: 1811-1814». Mélanges de la Casa de Velázquez. 10, 315-355.

LINARES VALCÁRCEL, F. (1999). Representaciones teatrales en Albacete: 1901-1923: cartelera, compañías y valoración. Albacete: Instituto de Estudios Albacentenses «Don Juan Manuel» de la Excelentísima. Diputación de Albacete, con prólogo de José Romera. (Pueden consultarse, tesis y cartelera, en http://www.uned.es/centro-investigacion-SELITEN@T/estudios_sobre_teatro.html).

LORENZ, Ch. M. (1938). «Seventeenth Century Plays in Madrid from 1808-1818». Hispanic Review 6, 324-331.

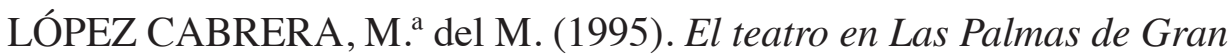
Canaria (1853-1900) (Tesis realizada bajo la dirección de José Romera y defendida en la UNED, enero de 1995. Pueden consultarse, tesis y cartelera, en http://www.uned.es/centro-investigacion-SELITEN@T/estudios_sobre_teatro.html. Extracto incluido en Aragón, 2006b).

LONDON, J. (1998).» Algunos montajes de Calderón en el Tercer Reich». En Texto e imagen en Calderón, Manfred Tietz (ed.), 143-157. Stuttgart: Franz Steiner.

MANCEBO SALVADOR, Y. (2002). «Hacia una historia de la puesta en escena de La vida es sueño». En Calderón en Europa, Javier Huerta Calvo, Emilio Peral Vega y Héctor Urzáiz Tortajada (eds.), 91-100. Madrid-Frankfurt am Main: Iberoamericana-Vervuert.

MONTERO DE LA FUENTE, L. (1942). «El teatro en Toledo durante el siglo XVIII (1762-1776)». Revista de Filología Española 26, 441468. 
MONFORTE DUPRET, R. (2008). «Recepción y escenificación del teatro clásico español en Rusia durante el siglo XX». Cuadernos de Teatro Clásico 24, 71-114.

McGAHA, M. D. (1979). The Theatre in Madrid during the Second Republic: a Cheklisk. London: Grant and Cutler.

MENÉNDEZ PELÁEZ, J. (1981). El teatro en Asturias (de la Edad Media al siglo XVIII). Gijón: Noega.

OCHANDO MADRIGAL, E. (2000). El teatro en Albacete durante la Edad de Plata (1924-1936). Albacete: Instituto de Estudios Albacentenses «Don Juan Manuel» de la Excelentísima Diputación de Albacete, con prólogo de José Romera. (Pueden consultarse, tesis y cartelera, en http://www.uned.es/centro-investigacion-SELITEN@T/estudios_sobre_teatro.html. Extracto incluido en Aragón, 2006b).

ORDAZ, L. (1946). El teatro en el Río de la Plata: Desde sus orígenes hasta nuestros días. Buenos Aires: Ed. Futuro, S.R.L.

PAR, A. (1929). «Representaciones teatrales en Barcelona durante el siglo XVIII». Boletín Academia Española 16, 326-46, $492-513$ y 594-614.

PINO, E. (1985). Historia del teatro en Málaga durante el siglo XIX. Málaga: Editorial Arguval.

REVERTE BERNAL, C. (2008). «El teatro clásico español en Hispanoamérica». Cuadernos de Teatro Clásico 24, 119-165.

RODRIGUES VIANNA PERES, L. (2003). «La vida es sueño en los tablados de Brasil». En Teatro calderoniano sobre el tablado, M. Tietz (ed.), 405-12. Stuttgart: Franz Steiner.

ROMERA CASTILLO, J. (2011). Pautas para la investigación del teatro español y sus puestas en escena. Madrid: UNED.

ROMERA CASTILLO, J. (ed.) (2000). «Sobre teatro de los años noventa». Signa. Revista de la Asociación Española de Semiótica 9, 93-210.

ROMERA CASTILLO, J. (coord.) (2003). «En torno a la semiótica teatral: sobre algunas compañías profesionales en diversas ciudades españolas [siglos XIX y XX]» Signa. Revista de la Asociación Española de Semiótica 12, 323- 546.

SABIK, K. (2000). «Las puestas en escena de la obra de Calderón en Polonia en el siglo XX». En Calderón en escena: siglo XX, J.M. ${ }^{a}$ Díez y Andrés Peláez (eds.), 211-18. Madrid: T. F. Artes Gráficas, D. L. 
SCHIZZANO MANDEL, A. (2003). «La vida es sueño: Nuevas interpretaciones escénicas». En Teatro calderoniano sobre el tablado, $\mathrm{M}$. Tietz (ed.), 443-54. Stuttgart: Franz Steiner.

SIMÓN DÍAZ, J. (1963). «Los clásicos españoles en la prensa diaria de Madrid (1830-1900)». Revista de Literatura 23, 209-240.

SIMÓN DÍAZ, J. (dir.) (1961). Cartelera teatral madrileña I: años 18301839. Madrid: Consejo Superior de Investigaciones Científicas.

SILIUNAS, V. (2000). «Calderón en Rusia y Unión Soviética en el Siglo X». En Calderón en escena: siglo XX, J.M. ${ }^{a}$ Díez y Andrés Peláez (eds.), 181-89. Madrid: T. F. Artes Gráficas, D. L.

SIRERA, J. Ll. (1986). «La infraestructura teatral valenciana». En Teatro y prácticas escénicas. II. La Comedia, J.L. Canet Vallés (coord.), 2649. London: Tamesis.

SOMALO FERNÁNDEZ, M. Á. (2004). El teatro en Logroño (19011950) (Tesis realizada bajo la dirección de Julián Bravo Vega y defendida en la Universidad de La Rioja, en 2004. Puede consultarse tesis en http://www.uned.es/centro-investigacion-SELITEN@T/estudios_sobre_teatro.html. Extracto incluido en Aragón, 2006b).

- (2006). «La actividad teatral en Logroño entre 1901 y 1950». Signa. Revista de la Asociación Española de Semiótica 15, 493-534.

SUÁREZ MUÑOZ, Á. (1994). El teatro en Badajoz, 1860-1886: cartelera y estudio. Madrid: Támesis. (Pueden consultarse, tesis - dirigida por José Romera - y cartelera, en http://www.uned.es/centro-investigacion-SELITEN@T/estudios_sobre_teatro.html)

SUBIRATS, R. (1977). «Contribution a l'ètablessiment dù repertoire thèâtral à la Cour de Philippe IV et de Charles II». Bulletin Hispanique 79, 3-4, 401-479.

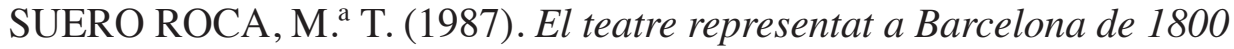
a 1830. Barcelona: Institut del Teatre.

SULLIVAN, H. W. (1998). Calderón alemán. Recepción de un genio hispano, 1654-1980. Madrid: Iberoamericana.

TORRES LARA, A. (1996). La escena toledana en la segunda mitad del siglo XIX (Tesis realizada bajo la dirección de José Romera y defendida en la UNED, septiembre de 1996. Pueden consultarse, tesis y cartelera, en http://www.uned.es/centro-investigacion-SELITEN@T/estudios_sobre_teatro.html) 
VAREY, J. E. y SHERGOLD, N. D. (1989). Comedias en Madrid, 16031709: repertorio y estudio bibliográfico. London: Tamesis Books, D.L.

ZABALA, A. (1982). El teatro en la Valencia de finales del XVIII. Valencia: Institución Alfons el Magnànim.

Recibido el 8 de febrero de 2012.

Aceptado el 30 de septiembre de 2012. 